\title{
Efeito analgésico e anti-inflamatório do extrato aquoso das folhas de trevo-roxo (Scutellaria agrestis A. St.-Hil. ex Benth. - Lamiaceae) em roedores
}

\author{
OLIVEIRA, A.B. ${ }^{*}$; BARBOSA, G.S. ${ }^{2}$; VERDAM, M.C. ${ }^{2}$; OHANA, D.T. ${ }^{2}$; MENDONÇA, M.S. ${ }^{3}$; \\ MEIRA, R.M.S.A. ${ }^{4}$ \\ ${ }^{1}$ Instituto Nacional de Pesquisas da Amazônia, INPA, Avenida André Araújo, 2936, Aleixo, 69060-001 Manaus- \\ Am, Brazil; '2Laboratório de Atividades Biológicas, Faculdade de Ciências Farmacêuticas (UFAM), Rua Alexandre \\ Amorim, 330, Aparecida, 69010-300 Manaus-Am, Brasil; ${ }^{3}$ Laboratório de Botânica Agroflorestal, Universidade \\ Federal do Amazonas (UFAM), Setor Sul, Av. General Rodrigo Otávio Jordão Ramos, 3000, Aleixo, 69077-000, \\ ${ }^{4}$ Laboratório de Anatomia Vegetal, Universidade Federal de Viçosa-UFV, Av. P. H. Rolfs, s/n Campus Universitário \\ Centro, 36560-000 Viçosa-MG, Brasil *andreiabo@hotmail.com
}

\begin{abstract}
RESUMO: Scutellaria agrestis é utilizada por comunidades ribeirinhas do Amazonas principalmente para o tratamento de otites por via tópica utilizando-se o extrato bruto obtido por maceração. O presente trabalho visou investigar preliminarmente o perfil fitoquímico, a segurança toxicológica e as ações analgésica, anti-inflamatória e antiedematogência do extrato aquoso das folhas de $S$. agrestis. Foram coletados 80 indivíduos da espécie no horto medicinal da Universidade Nilton Lins, Manaus, Brasil. O perfil fitoquímico foi obtido por meio de prospecção da droga vegetal para heterosídeos cianogênicos, terpenos, compostos fenólicos e alcaloides. A toxicologia foi avaliada pelo teste de toxicidade aguda. As atividades analgésicas/ anti-inflamatórias foram analisadas por meio dos testes de formalina em camundongos e a atividade antiedematogência, pelo teste de edema de pata em ratos. Os metabólitos detectados foram fenóis (taninos hidrolisáveis, cumarinas e várias classes de flavonoides) e terpenos (esteroides livres, saponinas). Não foi possível estabelecer $\mathrm{DL}_{50}$, haja visto que o extrato não provocou a morte de nenhum animal durante o teste de toxicidade aguda, provavelmente devido à ausência de heterosídeos cianogênicos na sua composição. Apesar de não provocar morte, considerou-se que o extrato apresenta uma discreta toxicidade, uma vez que foi observada a ocorrência de espasmos na primeira hora de observação dos animais. O extrato apresentou ainda efeito analgésico e anti-inflamatório significativo nas doses de 30,100 e $300 \mathrm{mg} / \mathrm{kg}$ pelo teste da formalina, sendo o resultado na maior dose equivalente ao obtido com a droga padrão (fentanil). No entanto, não observamos efeito antiedematogênico nas doses testadas durante as 5 horas de registro do edema de pata. Os resultados obtidos nesta pesquisa conferem base científica preliminar quanto à segurança e ao efeito analgésico e antiinflamatório da droga vegetal, o que indica que tal espécie é promissora e expressamente recomendada para maiores estudos farmacológicos in vitro e in vivo.
\end{abstract}

Palavras-chave: trevo-roxo, fitoquímica, ensaios biológicos.

ABSTRACT: Analgesic and anti-inflammatory effect of the aqueous extract of "trevo-roxo" (Scutellaria agrestis A. St.-Hil. ex Benth. - Lamiaceae) leaves in rodents. The Scutellaria agrestis is used by Amazonas riverine communities, especially for otitis externa topical treatment, by using the crude extract obtained by maceration. This study aimed to investigate the preliminary phytochemical profile, the safety/toxicity and the analgesic, anti-inflammatory and antiedematogenic activities of the aqueous extract of the $S$. agrestis leaves. Eighty individuals were collected at the Nilton Lins University medicinal garden, Manaus, Brazil. The phytochemical profile was obtained through a plant drug survey for cyanogenic heterosides, terpenes, alkaloids and phenolic compounds. The extract safety was evaluated by acute toxicity test. Analgesic and anti-inflammatory activities were accessed using formalin test in mice and the antiedematogenic activity, using paw edema test in mice. We detected phenolic (hydrolysable tannins, coumarins and several classes of flavonoids) and terpenoid (free steroids, saponins) metabolites. We could not establish $\mathrm{LD}_{50}$ because no animals died during the acute toxicity test, probably because of 
the absence of cyanogenic glycosides on the composition of the extract. However, we found that the extract is slightly toxic as animal spasms were observed in the first hour of the test. The extract showed significant analgesic and anti-inflammatory activity on the formalin test $(30,100$ and $300 \mathrm{mg} / \mathrm{kg}$ p.o.), and the highest dose result was equivalent to the standard drug (Fentanyl). However, no significant antiedematogenic effect was observed during the paw edema test. The results obtained in this study provide preliminary scientific basis about the safety and analgesic/ anti-inflammatory actions of the aqueous extract of $S$. agrestis, which indicates that this species is a promising option for further in vitro and in vivo pharmacological studies.

Keywords: "trevo-roxo", phytochemistry, biological assays.

\section{INTRODUÇÃO}

O gênero Scutellaria L. compreende aproximadamente 360 espécies de distribuição sub-cosmopolita (Paton, 1990; Pool, 2006). Dentre estas espécies destacam-se $S$. baicalensis, $S$. amoena, S. linearis, S. viscidula, S. strigillosa, S. prostrata, S. grossas, S. barbata, S. hypericifolia, S. galericulata, S. discolor, S. seleriana, S. ovata, $S$. linearis, $S$. rehderiana, como plantas medicinais muito utilizadas por povos tradicionais asiáticos, europeus e americanos no tratamento de diversas doenças.

A partir das aplicações registradas para tais espécies suas propriedades terapêuticas têm sido investigadas, tais como: atividades antiespasmódica, antifúngica, febrífuga, antioxidante, anticâncer, anti-HIV, antibactericida, antiviral, antiinflamatória, e anticonvulsiva (Ersöz et al., 2002; Chou et al., 2003; Lin et al., 2009; Shang et al., 2010; Huan et al., 2012). Ademais, cerca de 300 substâncias biologicamente ativas foram isoladas de 35 espécies de Scutellaria. Dentre estas, os dois principais grupos constituintes são os compostos fenólicos (flavonoides, glicosídeos feniletanoides) e os terpenos (glicosídeos iridoides, diterpenos e triterpenoides), muito embora também sejam encontrados nestas plantas outros metabólitos, tais como, os alcaloides, fitosteróis, polissacarídeos, entre outros (Shang et al., 2010).

O trevo-roxo (Scutellaria agrestis A. St.-Hil. ex Benth.) é uma planta cultivada por populações ribeirinhas do Amazonas, Brasil, e utilizada na medicina popular para fins terapêuticos de algumas doenças inflamatórias, principalmente otites, para as quais se utiliza por via tópica o extrato bruto obtido por maceração. Também é utilizada no tratamento de febre, diarréia e pressão alta, com uso do extrato aquoso obtido por infusão, administrado por via oral (Cassino, 2010).

Na medicina popular, é comum o uso de plantas medicinais para tratar otites, embora nem sempre tal uso seja respaldado cientificamente. A otite consiste na infecção do ouvido externo (inflamação da pele do canal auditivo externo) ou médio (inflamação da mucosa que reveste a cavidade timpânica). Ambas podem ser crônicas ou agudas, podendo a otite externa ser causada por bactérias e/ou fungos e a otite média, por bactérias e/ou vírus (Neto et al., 2006; Nogueira et al., 2008).

Diante da importância de avaliar a ação farmacológica de drogas vegetais, principalmente de plantas com informações restritas a dados etnofarmacológicos na região amazônica, como é o caso da espécie em questão, e ainda, considerando a importância do vasto potencial farmacológico reportado ao gênero Scutellaria (Shang et al., 2010), o presente trabalho visou investigar preliminarmente o perfil fitoquímico, a segurança toxicológica, e as ações analgésicas e/ou anti-inflamatórias do extrato aquoso das folhas de Scutellaria agrestis A. St.-Hil. ex Benth., com o propósito de dar início aos primeiros estudos da atividade terapêutica desta droga.

\section{MATERIAL E MÉTODOS}

Indivíduos cultivados de Scutellaria agrestis foram coletados as 10:00 horas da manhã do dia 05 de outubro de 2009, no Horto medicinal da Universidade Nilton Lins localizada na cidade de Manaus, Amazonas, Brasil (03 $3^{\prime} 37.5^{\prime \prime} \mathrm{S}$ e $60^{\circ}$ 00' 33.4"W) e imediatamente transportadas para o Laboratório de Farmacobotânica da Faculdade de Ciências Farmacêuticas da Universidade Federal do Amazonas (FCF/UFAM). Foram utilizados 30 indivíduos para a prospecção fitoquímica e 50 indivíduos para a realização dos ensaios farmacológicos. As amostras recém coletadas foram colocadas em estufa com circulação de ar interna a $40^{\circ} \mathrm{C} \pm 2{ }^{\circ} \mathrm{C}$ no período de 24 horas. Posteriormente, a partir do material seco, as folhas foram separadas manualmente e trituradas com auxílio de um moinho de faca.

Exsicatas foram confeccionadas, identificadas pelo Prof. Dr. Alan Paton (Kew Gardens), especialista do gênero Scutellaria, e incorporadas ao Herbário do Instituto Nacional de 
Pesquisa da Amazônia - INPA, sob o número de registro 232920.

\section{Prospecção fitoquímica preliminar}

O material vegetal seco e pulverizado (MVSP) foi utilizado para os seguintes testes de prospecção: heterosídeos cianogênicos, esteroides livres, triterpenoides pentacíclicos livres e compostos fenólicos tais como taninos hidrolisáveis, taninos condensados, antocianinas, antocianidinas, flavonoides, leucoantocianidinas, catequinas, flavonóis, flavonas, flavononóis e xantonas (Matos, 1997); saponinas (Schenkel et al., 2004); alcaloides (Costa, 2002) e cumarinas (Kuster \& Rocha, 2004).

\section{Preparação do extrato aquoso}

O extrato aquoso foi extraído por infusão a 5 $\%$ de folhas secas e trituradas de $S$. agrestis, durante 30 minutos, liofilizado em aparelho Christ Beta (modelo 1-8 LMC-1) e armazenado à temperatura ambiente, protegido da luz.

\section{Animais}

Foram usados 30 ratos Wistar (peso 200$250 \mathrm{~g}$ ), sendo quinze fêmeas e quinze machos, e 78 camundongos Swiss (peso 25-30 g), de ambos os sexos, metade de cada. Os animais foram mantidos em salas com temperatura de $22-25^{\circ} \mathrm{C}$, ciclo de luz $12 / 12 \mathrm{~h}$, com ração e água ad libitum. Os mesmos foram ambientados na sala de experimento durante 12 horas antes da realização dos ensaios. Os protocolos utilizados foram aprovados pelo Comitê de Ética de Experimentação Animal da Universidade Federal do Amazonas (CEEA-UFAM), sob o número 012/2010.

\section{Avaliação da toxicidade aguda e triagem hipocrática}

Para a avaliação da toxicidade aguda e triagem hipocrática (Brito, 1994), cinco grupos de seis camundongos receberam doses de 30 , $100,300,1000 \mathrm{mg} / \mathrm{kg} / \mathrm{dia}$ do extrato aquoso de $S$. agrestis por via oral através de gavagem, enquanto no grupo controle foi administrada água potável (10 $\mathrm{mL} / \mathrm{kg}$ ). Durante o experimento foram observadas alterações como parâmetros comportamentais e fisiológicos e mortalidade nas primeiras 5 horas e a cada 24 horas durante 15 dias.

\section{Avaliação da atividade analgésica e/ou anti- inflamatória pelo teste de formalina \\ Para o teste da formalina (Lapa et al., 2008) foram utilizados seis grupos de oito camundongos, nos quais foram administrados, por via oral, 10 $\mathrm{mg} / \mathrm{kg}$ de indometacina (Merck, Manaus, Brasil) solubilizada em solução $0,1 \mathrm{~N}$ de $\mathrm{NaOH}$ com pH 8 (controle positivo da fase anti-inflamatória), água}

potável, $10 \mathrm{~mL} / \mathrm{kg}$ (controle negativo) e extrato aquoso de $S$. agrestis nas concentrações 30,100 e $300 \mathrm{mg} / \mathrm{kg}$, e, por via subcutânea, $50 \mu \mathrm{g} / \mathrm{kg}$ de fentanil diluídos em solução salina $0,9 \%$ (controle positivo da fase analgésica). Após o período de uma hora, cada animal recebeu $30 \mu \mathrm{L}$ de solução de formalina $2 \%$ por via subplantar na pata direita. Em seguida, os animais foram observados nos primeiros 5 minutos, para avaliação da atividade analgésica (primeira fase), e dos 15 aos 30 minutos, para avaliação da ação anti-inflamatória (segunda fase). O tempo que cada animal passou batendo, lambendo ou mordendo a pata injetada foi registrado e as médias desses tempos observados nos grupos tratados foram comparadas à média do controle negativo.

\section{Avaliação da atividade anti-inflamatória pelo método de edema de pata em ratos}

Para o método de edema de pata em ratos (Lapa et al., 2008), foram utilizados cinco grupos com seis ratos, nos quais o edema foi induzido pela injeção de $0,3 \mathrm{~mL}$ de carragenina $(1,5 \%$ em salina estéril), administrada na região subplantar da pata direita de todos os animais. Na pata esquerda foram administrados $0,3 \mathrm{~mL}$ de salina, que corresponde ao controle individual dos animais.

Uma hora antes da injeção de carragenina, o extrato aquoso de $S$. agrestis foi administrado nas doses de 30, 100 e $300 \mathrm{mg} / \mathrm{kg}$ pela via oral. O grupo controle negativo recebeu $10 \mathrm{~mL} / \mathrm{kg}$ de água potável (v.o.). A indometacina (10 mg/kg, v.o., solubilizada em solução $0,1 \mathrm{~N}$ de $\mathrm{NaOH}$ com $\mathrm{pH}$ 8) foi usada como controle positivo do teste.

Após a aplicação da carragenina, a progressão do volume do edema foi mensurada com auxílio do aparelho hidropletismômetro digital (plethysmometer modelo LE 7500). Foram realizadas seis medidas, sendo uma basal e as demais a cada hora, durante cinco horas. As médias de cada grupo foram comparadas com o controle negativo, a cada tempo registrado.

\section{Análise estatística}

Os resultados foram avaliados em função da comparação com os grupos controle (água 10 $\mathrm{mL} / \mathrm{kg}$ ), analisados através de análise de variância (ANOVA), utilizando-se pós-teste de Dunnett's para 1 way e Bonferroni para 2way e expressos através da média do desvio padrão, sendo considerados significativos aqueles que apresentaram $P<0,05$. As análises estatísticas foram realizadas utilizando-se

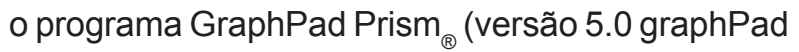
Software, Inc.) 


\section{RESULTADOS}

A prospecção fitoquímica preliminar obtida das folhas de Scutellaria agrestis registrou a presença de saponinas, esteroides livres, fenóis gerais, cumarinas, taninos hidrolisáveis e flavonoides como antocianinas, antocianidinas, chalconas, auronas, flavonas, flavonois, xantonas, leucoantocianidinas, flavanonas, flavanonóis (Tabela 1). Os componentes alcaloides, triterpenoides pentacíclicos livres e taninos condensados não foram detectados (Tabela 1).

No experimento da triagem hipocrática, referente aos parâmetros comportamentais, foi registrada a ocorrência de espasmos grosseiros na primeira hora de observação dos animais. Tais espasmos também foram observados nos demais experimentos realizados nesta pesquisa, ocorrendo logo após a administração oral das doses do extrato aquoso da droga testada.

Durante os 15 dias de observação referentes ao experimento de toxicidade aguda, não houve registro de morte de nenhum dos animais testados, portanto não foi possível determinar a $\mathrm{DL}_{50}$. O extrato foi considerado de baixa toxicidade no modelo testado, considerando-se que a dose testada (1000 mg/kg/dia) neste experimento foi a máxima, como o exigido pela Agência Nacional de Vigilância Sanitária (2010). Ademais, foi registrada por meio da prospecção fitoquímica a ausência de heterosídeos cianogênicos (Tabela 1) na folha de S. agrestis, corroborando os dados obtidos in vivo.
No teste de formalina, houve redução da resposta ao estímulo doloroso nas duas fases de observação (Figura 1). Nos primeiros cinco minutos do teste, que correspondem à fase neurogênica, observou-se uma redução média de cerca de $32 \%$ da sensação álgica nos animais que receberam as três doses do extrato quando comparados ao controle negativo, o que representa uma analgesia estatisticamente significativa $(P<0,05)$ em resposta ao estímulo nocivo quando comparado ao controle negativo. Durante a fase anti-inflamatória, avaliada nos últimos 15 minutos de observação, obteve-se redução média de $65 \%$ da resposta álgica nos animais tratados com as doses de 100 e 300 mg/ $\mathrm{kg}$ do extrato, sendo essa redução estatisticamente significativa $(P<0,05)$, quando comparada ao controle negativo. A resposta analgésica observada para a dose de $300 \mathrm{mg} / \mathrm{kg}$ do extrato foi equivalente àquela observada para a dose de $50 \mu \mathrm{g} / \mathrm{kg}$ de fentanil em ambas as fases de observação, não havendo diferença estatisticamente significativa entre esses tratamentos. Especificamente na segunda fase de observação, as doses de 100 e $300 \mathrm{mg} / \mathrm{kg}$ do extrato demonstraram atividade anti-inflamatória equivalente à droga padrão indometacina $(10 \mathrm{mg} /$ $\mathrm{kg}$ ), não sendo observada diferença estatísticamente significativa entre as mesmas.

TABELA 1. Prospecção fitoquímica da droga vegetal de Scutellaria agrestis A. St.-Hil. ex Benth.

\begin{tabular}{ll}
\hline Metabólitos & Folha \\
\hline Heterosídeos cianogênicos & - \\
Alcaloides & - \\
Saponinas & ++ \\
Esteroides livres & ++ \\
Triterpenoides pentacíclicos livres & - \\
Cumarinas & ++ \\
Fenóis gerais & ++ \\
Taninos hidrolisáveis & ++ \\
Taninos condensados & - \\
Antocianinas e Antocianidinas & ++ \\
Chalconas e Auronas & ++ \\
Flavonas, Flavonóis e Xantonas & ++ \\
Flavanonóis & - \\
Leucoantocianidinas & ++ \\
Catequinas (Taninos catéquicos) & - \\
Flavanonas & - \\
Flavonóis, Flavanonas, Flavanonóis e Xantonas & ++ \\
\hline
\end{tabular}

Reação positiva: +; Reação positiva forte: ++; Reação negativa: -.

Rev. Bras. PI. Med., Campinas, v.16, n.2, p.174-181, 2014. 


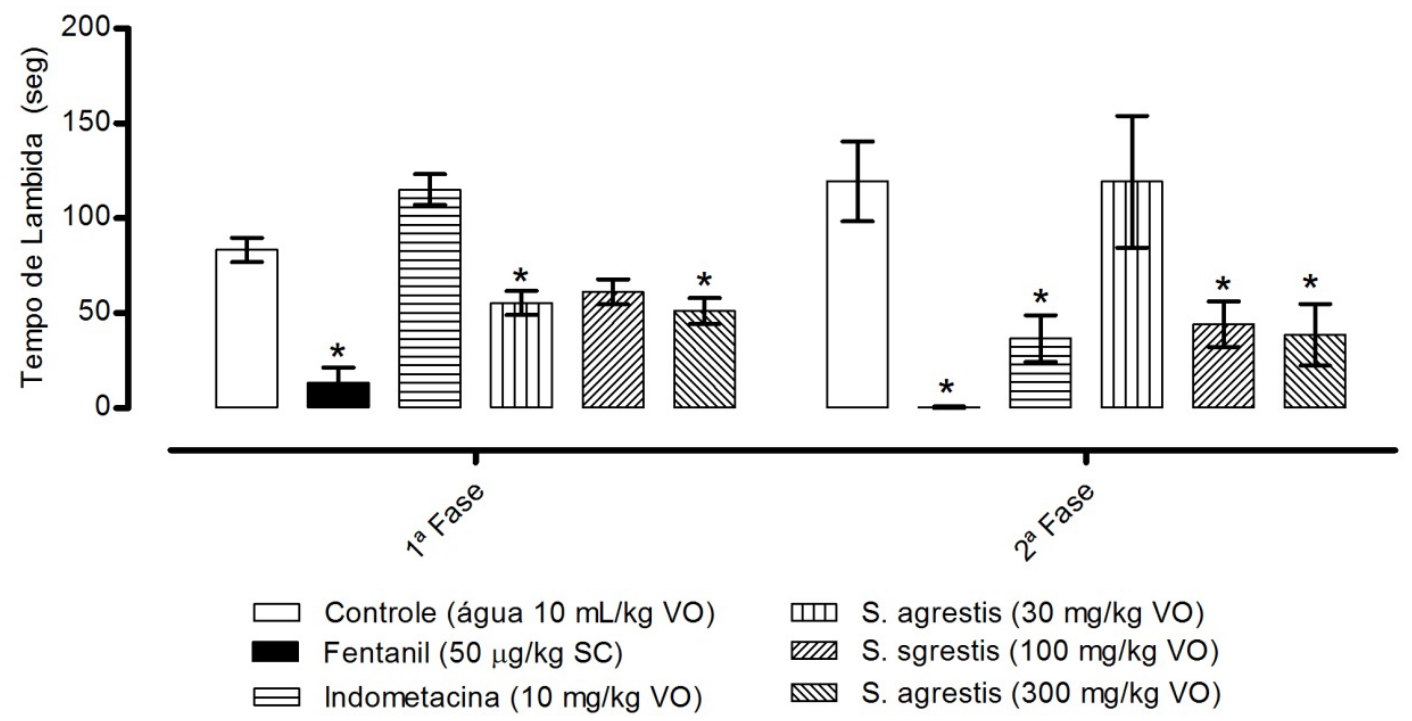

FIGURA 1. Atividade analgésica ( $1^{\mathrm{a}}$ fase) e anti-inflamatória ( $2^{\mathrm{a}}$ fase) do extrato aquoso de $S$. agrestis A. St.-Hil. Ex Benth. (30, 100 e $300 \mathrm{mg} / \mathrm{kg} \mathrm{VO}$ ), indometacina (10 mg/kg VO), água potável (10 mL/kg VO) e fentanil (50 $\mu \mathrm{g} / \mathrm{kg} \mathrm{SC}$ ) em camundongos $(\mathrm{n}=8)$ pelo teste de formalina. Dados expressos através da média \pm desvio padrão. ${ }^{*} \mathrm{P}<0,05$ versus controle; ${ }^{\mathrm{P}}>0,05$ versus fentanil. (ANOVA 2 way, pós-teste de Bonferroni).

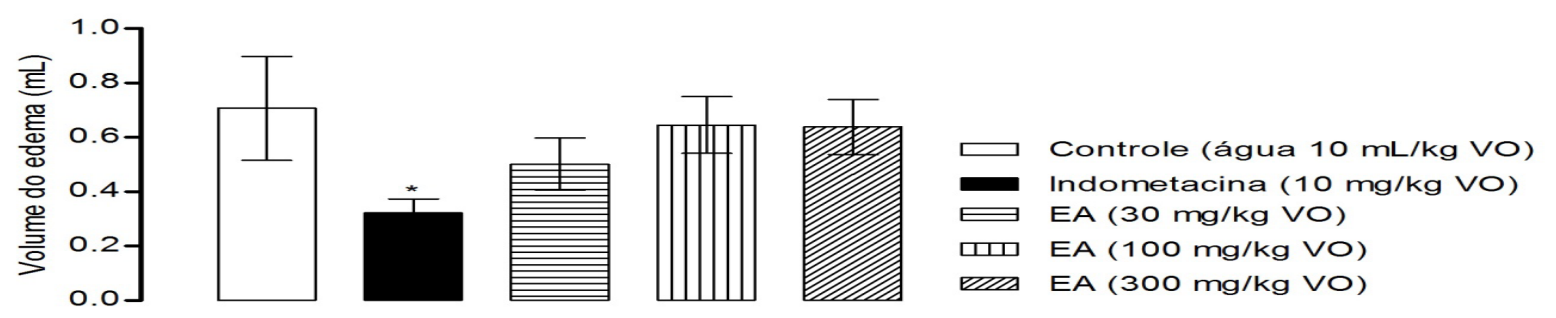

FIGURA 2. Atividade antiedematogênica do extrato aquoso de S. agrestis A. St.-Hil. Ex Benth. (30, 100 e 300 $\mathrm{mg} / \mathrm{kg} \mathrm{VO}$ ), indometacina (10 $\mathrm{mg} / \mathrm{kg} \mathrm{VO}$ ) e água potável $(10 \mathrm{~mL} / \mathrm{kg} \mathrm{VO})$ em ratos $(\mathrm{n}=6)$ através do teste de edema de pata na terceira hora de registro. Dados expressos através da média \pm desvio padrão. ${ }^{*} \mathrm{P}<0,05$ versus controle. (ANOVA 1way, pós-teste de Dunnett).

Naavaliação daatividadeantiedematogênica, pelo modelo de edema de pata em ratos, o extrato aquoso da folha de $S$. agrestis não demonstrou atividade estatisticamente significativa $(P>0,05)$ quando comparado ao controle negativo (Figura 2).

\section{DISCUSSÃO}

Em Scutellaria agrestis foi registrada a presença de terpenos e fenóis (flavonoides). Tais compostos geralmente estão presentes em várias espécies do gênero Scutellaria $(S$. linearis, $S$. baicalensis, S. amoena, S. linearis, S. viscidula, S. barbata, S. hypericifolia, S. galericulata, S. discolor), incluindo a presença de alcaloides em $S$. barbata e S. baicalensis (Hirotani et al., 1997; Bosabalidis et al., 1998; Hussain et al., 2008; Lin et al., 2009; Shang et al., 2010; Lee et al., 2011). Os fenóis e os terpenos, principalmente os flavonoides, e seus derivados (cerca de 160 compostos) como flavonas, flavonois, flavanonas, entre outros, já foram isolados de 35 espécies de Scutellaria e relacionados a várias atividades terapêuticas, como anti-inflamatória, anticâncer, antioxidante, antimutagênica, entre outras atividades (Shang et al., 2010; Park et al., 2011).

Neste contexto, são citados alguns exemplos de atividades terapêuticas relacionadas a estes compostos como a presença de flavonoides (baiacalina, baiacaleina) em Scutellaria baicalensis, relacionada às atividades de melhor desempenho da função contrátil cardíaca por meio de seus efeitos anti-inflamatórios, antioxidantes, antiapoptóticos, ainda contribuindo para os efeitos cardioprotetores e para aliviar as respostas ao estresse (Lee et al., 2007; Lee et al., 2011). Em Scutellaria 
barbata, foi verificado um grande número de flavonoides, alcaloides, esteroides, diterpenoides e polissacarídeos relacionados a efeitos inibitórios de cânceres humanos incluindo leucemia, hepatoma, câncer de colo de útero, de mama, do sistema digestivo e de fígado; ainda a partir do extrato bruto desta planta, foram testadas in vitro e in vivo as atividades antitumorais, tendo como resposta a não proliferação de células in vitro, inibindo o crescimento de tumores em roedores (in vivo) e ainda melhorando a função imunológica dos mesmos (Dai et al., 2011).

Entre as diversas atividades terapêuticas listadas para os representantes de Scutellaria, a atividade anti-inflamatória parece ser o efeito farmacológico característico do gênero (Shang et al., 2010; Lee et al., 2011; Huan et al., 2012). A inflamação é uma resposta do organismo vivo a uma agressão sofrida, caracterizada pelo surgimento dos sinais clássicos de rubor (eritema), calor (aumento da temperatura na região inflamada), tumor (edema), dor e perda de função do tecido ou órgão afetado (Falção et al., 2005). Há diferentes causas para o desenvolvimento do processo inflamatório, mas os mecanismos são comuns a todos os tipos (Falção et al., 2005).

O extrato aquoso de $S$. agrestis demonstrou uma ação acentuada sobre dois desses sinais da inflamação, a resposta nociceptiva (dor), apresentando um efeito analgésico nos animais já na primeira fase de observação do teste de formalina. O modelo experimental utilizado nesta pesquisa compõe-se de duas fases de resposta à formalina com mediação química e mecanismos de modulação distintas, apresentando diferenças marcantes quanto à sua sensibilidade a drogas analgésicas. Assim, a primeira fase refere-se a um caráter neurogênico, sendo sensível a analgésicos opioides e a alguns agonistas das vias descendentes, enquanto a segunda fase é caracterizada como dor de origem inflamatória, sendo sensível a analgésicos anti-inflamatórios não esteroidais (Lapa et al., 2008). Assim, pode-se interpretar que o extrato de $S$. agrestis possivelmente possua atividade do tipo opioide causando a dessensibilização das fibras periféricas responsáveis pela aferência dos sinais álgicos, o que não descarta inicialmente a possibilidade de efeito anti-inflamatório coexistente. Além disso, o registro dos animais apresentarem espasmos grosseiros, observados sempre na primeira hora após a administração das doses da droga testada, pode ser interpretado como a ocorrência de euforia. Tal evento comumente é propiciado por analgésicos do tipo opioide (Rang et al., 2004), corroborando o pressuposto para a droga estudada neste trabalho.

O termo opioide se aplica a qualquer substância, endógena ou sintética, que produz efeitos semelhantes à morfina (Rang et al., 2004). O ópio é um extrato obtido da seiva da papoula (Papaver somniferum), que contém dois tipos de alcaloides analgésicos, a morfina e a codeína (Rang et al., 2004; Heinz et al., 2010). A morfina, o principal componente, exerce ações depressoras e estimulantes em sítios específicos no sistema nervoso central (SNC), podendo apresentar efeitos como analgesia, euforia, sedação, depressão respiratória, supressão da tosse entre outros (Rang et al., 2004; Tripathi, 2006).

Como descrito, o alcaloide é a principal substância responsável pela ação analgésica do tipo opioide. Na espécie estudada, não foi detectada a presença deste composto, entretanto foi registrada a presença de terpenos. O diterpeno neoclerodane (salvinorina A), presente em Salvia divinorum (Lamiaceae), foi descoberto como um receptor opioide potente e seletivo (KOR) agonista, publicado como a primeira droga opioide não nitrogenada, ou seja, não alcaloide subtipo seletivo (Roth et al., 2002; Yan \& Roth, 2004). Salvinorina A possui propriedades psicoativas e, quando injetada, distribui-se por todo o cérebro, principalmente no cerebelo e córtex, com absorção extremamente rápida e curta duração e estima-se que menos de $10 \mu \mathrm{g}$ no cérebro podem ser responsáveis pelos efeitos alucinógenos (Hooker et al., 2008). Após a descoberta de salvinorina A, estão sendo desenvolvidos alguns estudos voltados para a síntese de sintéticos deste diterpeno (Simpson et al., 2009; McGovern et al., 2010), assim como para a sua utilização como uma droga potencial ansiolítica e antidepressiva (Braida et al., 2009).

Diante do exposto, há necessidade de maiores investigações quanto à substância responsável pela ação farmacológica da droga (S. agrestis) testada neste estudo, uma vez que há referências literárias de vários diterpenos neoclerodane para o gênero, tais como diterpenoides neoclerodanes jodrellin $B$, jodrellin $A$, scutalbin $A$, scutecyprol B isolados de Scutellaria rubicunda subsp. linneana (Carbel) Rech., S. rubicunda subsp. rubicunda Hornem e diterpenoides hastifolins A-C isolados de S. hastifolia (Bruno et al., 1999; Bruno et al., 2002; Raccuglia et al., 2010). Ainda, vale ressaltar a indicação do extrato de Scutellaria baicalensis e $S$. lateriflora para a redução da ansiedade, depressão, insônia, estresse, irritabilidade e inquietação (Lee et al., 2007; Head \& Kelly, 2009).

Avaliando-se o extrato de Scutellaria agrestis durante a segunda fase de observação do teste de formalina (anti-inflamatória), obteve-se uma redução média de $65 \%$ da resposta álgica nos animais tratados com as doses de 100 e 300 $\mathrm{mg} / \mathrm{kg}$ do extrato. A dor inflamatória é ocasionada 
pela liberação de mediadores inflamatórios que desencadeiam a sensibilização de nociceptores presentes nas terminações de fibras nervosas aferentes primárias, tendo como consequência um desencadeamento de respostas maiores em intensidade e/ou duração (Rang et al., 2004).

A atividade antiedematogênica realizada a partir do extrato aquoso da folha de $S$. agrestis mostrou resultados não significativos, verificandose que o extrato desta planta não tem efeito na redução do edema durante o processo inflamatório, apresentando apenas ação analgésica registrada no teste de formalina. Rang et al. (2004) reportam que a dor associada à lesão em neurônios da via nociceptiva, em vez de estímulo periférico excessivo, é frequentemente um componente dos estados da dor crônica e pode responder mal aos analgésicos opiáceos. Ademais, quando uma droga vegetal apresenta efeito antinociceptivo acessado pelo modelo das contorções abdominais ou pelo teste de formalina (segunda fase) dissociado da ação antiedematogênica no modelo da carragenina; podese, então, descartar a possibilidade desta droga estar alterando a participação das prostaglandinas no processo anti-inflamatório (Lapa et al., 2008). Diante dos efeitos farmacológicos obtidos para S. agrestis, possivelmente sua ação não esta relacionada às prostaglandinas, contudo são necessários estudos in vitro para se esclarecer o mecanismo de ação desta droga.

Neste contexto, a partir do extrato obtido da raiz de Scutellaria baicalensis registrou-se um potente efeito analgésico pautado a outros mecanismos anti-inflamatórios no qual foi proposto que os mecanismos de Baicalina (substância isolada do extrato) podem estar associados à inibição de mediadores inflamatórios (citocinas pró-inflamatórias como a PGE2) e ainda, outras possibilidades como um aumento de IL-10 e a inibição da infiltração de neutrófilos induzida pela injeção de carragenina nas patas dos animais. Assim, sugere-se que Baicalina pode ser terapeuticamente útil para mitigar a dor inflamatória (Chou et al., 2003; Yoon et al., 2009).

$O$ extrato aquoso de $S$. agrestis nos experimentos fitoquímicos e de toxicidade in vivo no modelo testado apresentaram-se de baixa toxicidade. Os flavonoides geralmente são substâncias que possuem baixa toxicidade do ponto de vista farmacológico e numerosos efeitos biológicos e atividades terapêuticas (Harborne \& Williams, 2000). Tendo em vista que a droga estudada apresenta muitos tipos de flavonoides, é possível relacionar a presença destas substâncias à baixa toxicidade registrada neste estudo. Ainda vale ressaltar que a baixa toxicidade é considerada uma propriedade importante para a seleção de compostos promissores (Pereira, 2007).

\section{CONCLUSÕES}

Os dados obtidos nesta pesquisa fornecem base científica preliminar para o uso popular do extrato aquoso de $S$. agrestis pela comunidade ribeirinha do Amazonas. Entretanto, sugerem-se mais experimentos in vivo e in vitro com intuito de estudar o mecanismo de ação analgésica da droga e investigação de outras propriedades terapêuticas. A baixa expressão dos sinais de toxicidade em animais de experimentação expostos a altas doses do extrato proporciona uma margem de segurança confortável para os avanços dos estudos in vivo com esta espécie.

\section{AGRADECIMENTOS}

À Faculdade de Ciências Farmacêuticas da Universidade Federal do Amazonas, pelo apoio durante realização dos experimentos fitoquímicos e farmacológicos; e a Capes pela concessão da bolsa.

\section{REFERÊNCIA}

BOSABALIDIS, A.; GABRIELI, C.; NIOPAS, I. Flavone aglycones in glandular hairs of Origanum $x$ intercedens. Phytochemistry, v.49, n.6, p.1549-53, 1998.

BRAIDA, D. et al. Potential anxiolytic- and antidepressantlike effects of salvinorin $A$, the main active ingredient of Salvia divinorum, in rodents. British Journal of Pharmacology, v.157, p.844-53, 2009.

BRITO, A.S. Manual de ensaios toxicológicos in vivo. 1. ed. Campinas: Editora da Unicamp, 1994. 124p.

BRUNO, M; VASSALLO, N.; SIMMONDS, M.S.J. A diterpenoid with antifeedant activity from Scutellaria rubicund. Phytochemistry, v.50, p.973-6, 1999.

BRUNO, M. et al. Simmonds, M.S.J. Antifeedant activity of neo-clerodane diterpenoids from two Sicilian species of Scutellaria. Biochemical Systematics and Ecology, v.30, p.793-9, 2002.

CASSINO, M.F. Estudo etnobotânico de plantas medicinais em comunidades de várzea do rio Solimões, Amazonas e aspectos farmacognósticos de Justicia pectoralis Jacq. forma mutuquinha (Acanthaceae). 2010. 135p. Dissertação (Mestrado - Área de Concentração em Botânica) - Programa de Pós-graduação em Botânica, Instituto Nacional de Pesquisas da Amazônia, Amazonas.

$\mathrm{CHOU}, \mathrm{T} . \mathrm{C}$. et al. The anti-inflammatory and analgesic effects of Baicalin in carrageenan-evoked thermal hyperalgesia. Anesthesia \& Analgesia, v.97, p.1724 -29, 2003.

COSTA, A.F. Farmacognosia. 6. ed. Lisboa: Fundação Calouste Gulbenkian, 2002.1031p.

DAI, Z.J. et al. In Vitro and in Vivo antitumor activity of Scutellaria barbate extract on murine liver cancer. Molecules, v.16, p.4389-400, 2011.

ËRSÖZ, T. et al. Phenylethanoid Glycosides from Scutellaria Galericulata. Chemistry, v.26, p.465- 71, 2002.

FALCÃO, H.S. et al. Review of the plants with anti- 
inflammatory activity studied in Brazil. Brazilian Journal of Pharmacognosy, v.15, p.381-91, 2005.

HARBORNE, J.B.; WILLIAMS, C.A. Advances in flavonoid research since 1992. Phytochemistry, v.55, p.481-504, 2000.

HEAD, K.A.; KELLY, G.S. Nutrients and botanicals for treatments of stress: adrenal fatigue, neurotransmitter, imbalance, anxiety, and restless sleep. Alternative Medicine Review, v.14, n.2, p.114-40, 2009.

HEINZ, L.; MOHR, K.; HEIN, L. Farmacologia: texto e atlas. 6. ed. Porto Alegre: Artmed, 2010. 408p.

HIROTANI, Y.Z.M.; YOSHIKAWA, T.Y.; FURUYA, T. Flavonoids and phenylethanoids from hairy root cultures of Scutellaria baicalensis. Phytochemistry, v.44, n.1, p.83-7. 1997.

HOOKER, J.M. et al. Pharmacokinetics of the potent hallucinogen, salvinorin $A$ in primates parallels the rapid onset, short duration of effects in humans. Neuroimage, v.41, n.3, p.1044-50, 2008

HUAN, S.K.H. et al. Scutellaria baicalensis alleviates cantharidin-induced rat hemorrhagic cystitis through inhibition of cyclooxygenase-2 overexpression. Molecules, v.17, p.6277-89, 2012.

HUSSAIN, H. et al. Chemical constituents of Scutellaria linearis. Biochemical Systematics and Ecology, v.36, p.490-2, 2008.

KUSTER, R.M.; ROCHA, L.M. Cumarinas, Cromonas e Xantonas. In: SIMÕES, C.M.O. et al. Farmacognosia: da planta ao medicamento. 5. ed. Porto Alegre: Editora da Universidade Federal do Rio Grande do Sul, 2004. p.537-56.

LAPA, A.J. et al. Métodos de avaliação da atividade farmacológica de plantas medicinais. São Paulo: Editora da Universidade Federal de São Paulo, 2008. $144 p$.

LEE, S. et al. Schizandra chinensis and Scutellaria baicalensis counter stress behaviors in mice. Phytotherapy Research, v.21, p.1187-92, 2007.

LEE, Y.M. et al. Baicalein, an active component of Scutellaria baicalensis Georgi, improves cardiac contractile function in endotoxaemic rats via induction of heme oxygenase-1 and suppression of inflammatory responses. Journal of Ethnopharmacology, v.135, p.179-85, 2011.

LIN, L.; HARNLYA, J.M.; UPTON, R. Comparison of the Phenolic Component Profiles of Skullcap (Scutellaria lateriflora) and Germander (Teucrium canadense and T. chamaedrys), a Potentially Hepatotoxic Adulterant. Phytochemistry, v.20, p.298-306, 2009.

MATOS, F.J.A. Introdução a Fitoquímica Experimental. 1. ed. Fortaleza: Editora da Universidade Federal do Ceará, 1997.141p.

MCGOVERN, D.L. et al. CoMFA analyses of C-2 position salvinorin A analogs at the kappa-opioid receptor provides insights into epimer selectivity. Journal Mol Graph Model, v.28, n.7, p.612-25, 2010.

NETO, J.F.L.; HEMB, L.; SILVA, D.B. Fatores de risco para otite média aguda recorrente: onde podemos intervir? - uma revisão sistemática da literatura. Jornal de Pediatria, v.82, p.87-96, 2006.

NOGUEIRA, J.C.R.; DINIZ, M.F.M.; LIMA, E.O. Atividade antimicrobiana in vitro de produtos vegetais em otite externa aguda. Revista Brasileira Otorrinolaringol, v.74, n.1, p.118-24, 2008.

PARK, K. et al. Korean Scutellaria baicalensis water extract inhibits cell cycle G1/S transition by suppressing cyclin D1 expression and matrix-metalloproteinase-2 activity in human lung cancer cells. Journal of Ethnopharmacology, v.133, p.634-41, 2011.

PATON, A. A global taxonomic investigation of Scutellaria. Kew Bull, v.45, p.399-450, 1990.

PEREIRA, D.G. Importância do metabolismo no planejamento de fármacos. Química Nova, v.30, n.1, p.171-7, 2007.

POOL, A. New Species of Scutellaria (Lamiaceae) from Mesoamerica. Novon, v.16, n.3, p.388-403, 2006.

RACCUGLIA, R.A. et al. Simmonds, M.S.J. Hastifolins A-G, antifeedant neo-clerodane diterpenoids from Scutellaria hastifolia. Phytochemistry, v.71, p.208791, 2010.

RANG, H.P. et al. Farmacologia. 5. ed. Rio de Janeiro: Elservier, 2004. 904p.

ROTH, B.L. et al. Salvinorin A: a potent naturally occurring nonnitrogenous $\mathrm{k}$ opioid selective agonist. Neurobiology, v.99, n.18, p.11934-9, 2002.

SCHENKEL, E.P.; GOSMANN, G.; ATHAYDE, M.L. Saponinas. In: SIMÕES, C.M.O. et al. Farmacognosia: da planta ao medicamento. 5. ed. Porto Alegre: Editora da Universidade Federal do Rio Grande do Sul, 2004. p.711-40.

SHANG, $X$. et al. The genus Scutellaria an ethnopharmacological and phytochemical review. Journal of Ethnopharmacology, v.128, p.279-313, 2010.

SIMPSON, D.S. et al. Synthetic studies of neoclerodane diterpenes from Salvia divinorum: role of the furan in affinity for opioid receptors. Org Biomol Chemistry, v.7, n.18, p.3748-56, 2009.

TRIPATHI, K.D. Farmacologia médica. 5. ed. Rio de Janeiro: Guanabara Koogan, 2006. 755p.

YAN, F.; ROTH, B.L. Salvinorin A: A novel and highly selective n-opioid receptor agonist. Life Sciences, v.75, p.2615-19, 2004.

YOON, S.B. et al. Anti-inflammatory effects of Scutellaria baicalensis water extract on LPS-activated RAW264.7 macrophages. Journal of Ethnopharmacology, v.125, p.286-90, 2009. 